of the same medium which are at different temperatures. ${ }^{5}$ The account of the investigations is illustrated by a number of excellent photographs showing the rolling up of the surface of discontinuity into vortices, and the separate convolutions can easily be seen. There are also several tables of numerical results which can be used when we have a complete theory capable of explaining the rolling up and of giving the value of the dominant wave-length or wave-lengths.

Previous investigators, theoretical and experimental, seem to have concentrated on discussing the surface separating two streams of equal temperature but differing velocities. Banerji and Ghatage supplement the existing information by investigating the effect of a difference in temperature in addition to the difference in velocity. The results, some of which might have been expected on general grounds, are interesting. A discontinuity in temperature alone, and hence a discontinuity in density, was introduced by making a cylindrical or rectangular column of water in a large tank differ in temperature from the adjoining fluid. Discontinuities in velocity as well as in density were produced by making two streams of water meet at various angles under different therma] conditions.

In all cases the initial surface of separation was in a vertical plane and not, as in most previous work, in a horizontal plane. This makes the basic motion unsteady, so that the surface of discontinuity has a tendency to change its position quite apart from its inherent instability. The instability, however, was quite apparent. The surface assumed a wave pattern, each crest or trough took up the form of a breaker and rolled round itself, thus producing a vortex and a series of spiral-shaped three-dimensional vortices was formed. "In the direction of motion the vortices showed a progressive increase in dimensions and number of convolutions. The greater the difference of temperature at the surface of discontinuity, or the greater its slope, the smaller was the diameter of the vortices. For very small difference of temperature the vortices had enormous diameters. The greater the difference of temperature at the surface of discontinuity, the more rapidly did the vortices develop." The shape of the surface of discontinuity was obtained by colouring various parts of the liquid and by photographing the visible stream lines in horizontal and vertical sections. The paths of the particles at the surface of discontinuity were also photographed but the actual material used to make the motion visible probably has some small influence on the paths of the particles of fluid.

L. ROSEnHead.

${ }^{1}$ Helmholtz, Phil. Mag., 36 ; 1868.

2 Kelvin, Phil. Mag., 42; 1871.

${ }^{3}$ Rayleigh, Proc. Lomdon Math. Soc., 10; 1879.

- Rosenhead, Proc Roy Soc. A 134 . 1931.

s Banerji and Ghatage, Indian J. Phys., 7 ; 1932.

\title{
Population Density and Egg-Laying in Flies
}

N a paper of much interest, Prof. Raymond Pearl has made a study of the influence of density of population upon egg production in Drosophila ( $J$. Exp. Zool., vol. 63, No. 1), which is a continuation of his investigations on the biological relations underlying the growth of populations.

The flies were placed under standard conditions and deposited their eggs on an agar surface of fixed area where they could be counted. In general, the higher the number of pairs of flies in a bottle of given size the smaller the number of eggs laid per individual. This result, obtained with different populations of flies, was then repeated by comparing the egg production of the same flies when kept for alternating twenty-hour periods under conditions of high or low population density. More than 100,000 eggs were counted in these experiments.

The decrease in the rate of egg production with increasing density of population is described by the same type of mathematical equation as that which relates the density of a gas to the mean free path of its molecules. From numerous observations of the behaviour of single flies under isolated conditions, they are found to go through regular cycles of feeding, walking or flying, resting and cleaning their legs. These rhythms are easily disturbed and the flies are found to be extremely sensitive to slight environmental disturbances. The denser the population the more they interfere with each other's rhythmic activities, thus decreasing the amount of food intake and oviposition and increasing their muscular activity. That the area of agar surface for feeding and egglaying, and not the volume of air, is the significant factor, was shown by comparing the oviposition in bottles of twice the size but with the same area of agar.

In the same journal, Dr. Alpatov has investigated egg production in vestigial- and long-winged flies under different conditions of development, the former showing much lower productivity. The influence of temperature and underfeeding in the larval stage were investigated. At a low temperature, egg production begins earlier. Underfeeding reduces productivity, and a negative correlation is found between length of life and egg production.

\section{Mycorrhiza on Conifer Roots}

A VERY thorough study of the root system of young conifers has been made by Dr. E. V. Laing for the Forestry Commission, and an account of this work is published by the Commission as Bulletin 13 under the title of "Studies on Tree Roots" (1932). This account contains an interesting contribution to our knowledge of mycorrhiza on these roots.

There are two forms of mycorrhiza; the ectotrophic, in which the fungus grows mainly on the surface of the root, and the endotrophic, in which it penetrates the cells of the cortex practically as far inwards as the endodermis. Dr. Laing points out that whilst both forms may occur on the same tree yet, on the whole, the ectotrophic type is common in pine, spruce and lareh, whilst the endotrophic type is characteristic of Cupressus, Thuya and Taxus. This distribution of the fungus correlates in an interesting manner with differences in cortical structure. In the first group of conifers the cortical cells have thin walls and contain little starch; in the second group characteristic girdle thickenings occur on the walls of the cortical cells and these cells usually contain starch; this is the type of cortex which is usually invaded by the fungus. 